\title{
Sexual selection
}

\section{Are ducks impressed by drakes' display?}

urprisingly few birds have penises, but among those that do, the Argentine lake duck (Oxyura vittata) tops the bill - the penis of this small stifftail duck from South America is shaped like a corkscrew and, at almost half a metre long, is the largest of any bird measured so far. Factors responsible for the evolution of this remarkable organ could include runaway selection, whereby drakes with longer penises gain dominance and copulate with more females, or preference by females for drakes with longer and more decorated penises.

This small duck (which typically weighs around $640 \mathrm{~g}$ ) was previously reported to have a $20-\mathrm{cm}$ penis ${ }^{1,2}$, which is about half of the drake's total body length and is comparable to the penis of an ostrich (Struthio camelus) ${ }^{3}$. A new voucher specimen from Argentina's Córdoba province (Fig. 1) indicates, however, that the penis size of this species was underestimated. We measured the length of the unwound, everted penis of an aroused $O$. vittata drake and found that it was $32.5 \mathrm{~cm}$ long when hanging under the force of gravity, but that it could stretch

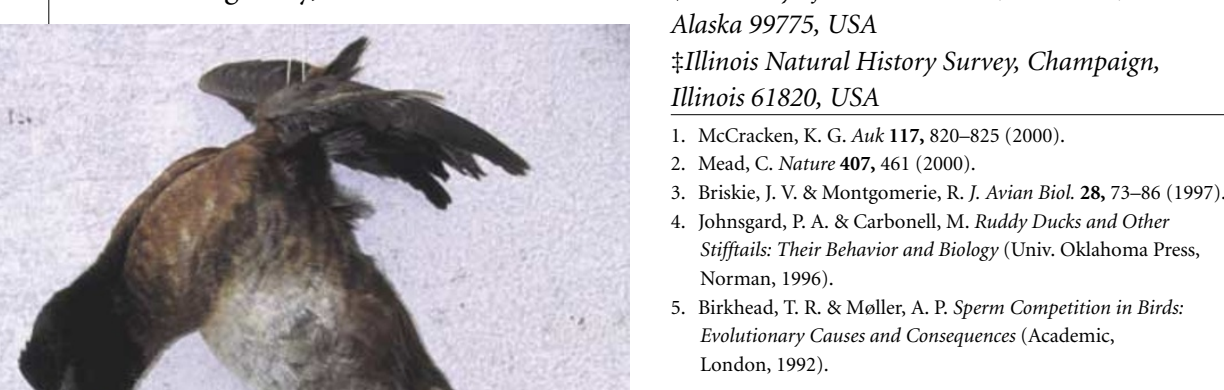

hen fully unwound to $42.5 \mathrm{~cm}$.

Like other stifftail ducks, lake ducks are promiscuous and boisterous in their sexual activity ${ }^{4}$. One explanation for the evolution of such a long penis might be selection for its competitive advantage - for instance, groups of drakes might display their everted penises to attract females. Success in sperm competition $^{5}$ may also be a factor. The base of the lake-duck penis is covered with spines, yet the tip is soft and brush-like. Before ejaculation, drakes probably use their penises like bottle-brushes to remove sperm stored in the oviduct by the female's previous consort. The larger the bottle-brush, the more effective it will be, clearing the way for the drake to father the ducklings.

Many questions remain unanswered. How much of his penis does the drake actually insert, and does the anatomy of the female's oviducts make them unusually difficult to inseminate? The Argentine lake duck offers a sizeable opportunity to study sexual selection and sperm competition in birds.

Kevin G. McCracken ${ }^{\star}$, Robert E. Wilson ${ }^{\star}$, Pamela J. McCracken $\dagger$, Kevin P. Johnson $\ddagger$ ${ }^{*}$ Institute of Arctic Biology and Department of Biology and Wildlife, University of Alaska

Fairbanks, Fairbanks, Alaska 99775, USA

e-mail:fnkgm@uaf.edu

$\dagger$ University of Alaska Museum, Fairbanks,

Alaska 99775, USA

¥Illinois Natural History Survey, Champaign,

61820, USA

C. Nature 407, 461 (2000).

Norman, 1996)

volutionary Causes and Consequences (Academic,

London, 1992).

\section{Nitrogen fixation}

\section{Endocrine disrupters and flavonoid signalling}

itrogen fixation is a symbiotic process initiated by chemical signals from legumes that are recognized by soil bacteria. Here we show that some endocrine-disrupting chemicals (EDCs) ${ }^{1-3}$, so called because of their effect on hormone-signalling pathways in animal cells, also interfere with the symbiotic signalling that leads to nitrogen fixation. Our results raise the possibility that these phytochemically activated pathways may have features in common with hormonal signalling in vertebrates, thereby extending the biological and ecological impact of EDCs.

Initiation and maintenance of nitrogenfixing symbiosis between rhizobial bacteria (Sinorhizobium meliloti) and alfalfa (Medicago sativa) requires an exchange of signals between these organisms ${ }^{4}$. In S. meliloti, the constitutively expressed product of the nodD genes acts as a positive regulator which, upon recognizing agonistic phytochemicals produced by alfalfa, activates transcription of the common nodulation (nod) genes needed to initiate symbiosis ${ }^{4,5}$. Whereas some flavonoids, such as luteolin and apigenin, stimulate nodD activation, others (such as chrysin) can antagonize the induction of nod genes ${ }^{5-7}$. Chemicals in the environment that mimic or interfere with this phytochemical signalling to $S$. meliloti may confound recognition crucial to symbiosis.

As luteolin and other flavonoids have been proposed to mimic oestrogen signalling in mammalian cells ${ }^{2}$ and a possible homology has been identified between NodD and the oestrogen receptor ER- $\alpha$ (ref. 8), we investigated whether EDCs that disrupt oestrogen signalling could also interfere with symbiotic signalling ${ }^{3}$. We tested the effects in S. meliloti of different compounds, added in the presence of luteolin, on activation and transcription of a nod reporter gene using a $\beta$-galactosidase assay ${ }^{9}$.

The inhibitory flavonoids chrysin and genistein $^{7,10}$ were among the most potent phytochemical-NodD signalling inhibitors, as were the organochlorine pesticides pentachlorophenol and methyl parathion, which each reduced nod expression by up to $90 \%$ even in the presence of luteolin (Fig. 1a) or apigenin (results not shown).

Half-maximal inhibitory concentrations $\left(\mathrm{IC}_{50}\right)$ were comparable for the inhibitory phytochemicals and the EDCs tested (Fig. 1a). Even nanomolar concentrations of some pesticides significantly inhibited phytochemical-NodD signalling (Fig. 1a). The insecticide dichlorodiphenyltrichloroethane (DDT) and its metabolites inhibited nod expression by an average of $40 \%$ (see, for example, Fig. 1b); other $\mathrm{EDCs}^{3}$, including the plasticizer bisphenol A (Fig. 1a) and the herbicides 2,4-D and 2,4,5-T (results not shown), reduced expression by $66 \%$, $32 \%$ and $37 \%$, respectively. Endosulfan, methoxychlor, aldrin, dieldrin, dursban, vinclozolin and diazinon had no effect on symbiotic signalling (results not shown).

The inhibitory EDCs are all planar phenolic compounds, whereas those that had no effect in our assay are non-planar and have no free hydroxyl group. Inhibition of transcription regulated by the luteolin-NodD pathway can be overcome by increasing the concentration of luteolin (Fig. 1b).

We grew alfalfa seedlings inoculated with the bacterial reporter strain on medium containing X-gal ${ }^{11,12}$; NodD-initiated gene transcription produces $\beta$-galactosidase which, in the presence of $\mathrm{X}$-gal, generates a blue prod$\mathrm{uct}^{11}$. Figure $1 \mathrm{c}-\mathrm{f}$ shows that $\beta$-galactosidase is expressed along the entire root system in the alfalfa control, whereas blue product is almost absent in roots treated with chrysin, lake duck (Oxyura vittata) taken near Arroyo Chucul, Córdoba, Argentina on 30 April 2001 

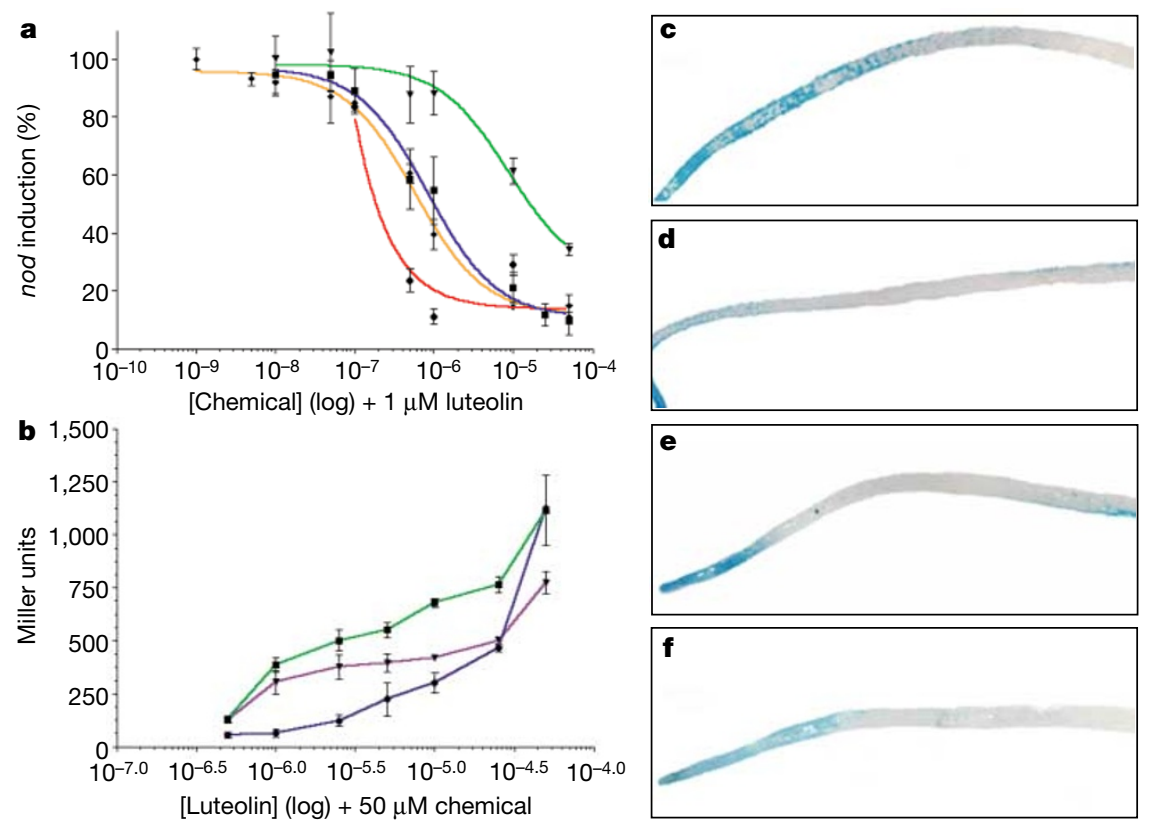

Figure 1 Endocrine-disrupting chemicals (EDCs) alter symbiotic signalling in vitro and in vivo. a, Phytochemicals (chrysin, orange) or EDCs (methyl parathion, green; pentachlorophenol, blue; bisphenol A, red) at $50 \mathrm{nM}$ to $50 \mu \mathrm{M}$ were added to Sinorhizobium melilot 1021pRmM57 (ref. 9; provided by S. R. Long) treated with $1 \mu \mathrm{M}$ luteolin. Luteolin alone was set as 100\% induction ${ }^{10}$; luteolin plus chemical was compared to $100 \%$ induction. $I_{50}$ values (molar): methyl parathion, $4.3 \times 10^{-7}$; pentachlorophenol, $9.9 \times 10^{-7}$; chrysin, $7.0 \times 10^{-7}$; bisphenol A, $1.7 \times 10^{-5}$. b, $50 \mu \mathrm{M}$ o, $p^{\prime}$-DDT (purple) or pentachlorophenol (blue) caused $43 \%$ and $90 \%$ inhibition, respectively, of nod activation (measured as Miller units of $\beta$-galactosidase activity). Increasing the luteolin concentration (100 nM to $50 \mu \mathrm{M}$ ) restores nod activation to normal. c-f, Alfalfa seedlings were grown on buffered nodulation medium plus X-gal ${ }^{11,12}$ and inoculated with dilute ${ }^{12}$ S. meliloti $1021 \mathrm{pRmM} 57$ treated with vehicle (dimethylsulphoxide, c), chrysin (d), methyl parathion (e) or pentachlorophenol (f) at $50 \mu \mathrm{M}$. NodC-lacZ expression is visualized as blue product. Further details are available from the authors.

pentachlorophenol or methyl parathion, except between the tip and the first emerging root hairs, where luteolin concentration is highest $^{6,10}$. This effect is similar to that shown in Fig. 1b, where inhibition is overcome by increasing concentrations of luteolin.

Nitrogen fixation is controlled by both agonistic and antagonistic phytochemical signalling to Rhizobium ${ }^{5,13}$. As with the vertebrate endocrine system, plant-bacterial symbiosis relies on recognition of specific agonists and antagonists for gene regulation $^{2,10,13}$. We have shown that some pesticides and planar phenolic EDCs can interfere with plant-Rhizobium signalling and nitrogen-fixing symbiosis, thereby exposing a previously unrecognized similarity to the effects of EDCs on vertebrate endocrine signalling. Jennifer E. Fox ${ }^{\star} \dagger$, Marta Starcevic ${ }^{\star}$, Kelvin Y. Kow ${ }^{\star}$, Matthew E. Burow ${ }^{\star} \ddagger$, John A. McLachlan ${ }^{\star}$

${ }^{\star}$ Environmental Endocrinology Laboratory, Center for Bioenvironmental Research at Tulane and Xavier Universities, $\uparrow$ Molecular and Cellular Biology

Program, Tulane University, and $\ddagger$ Department of Pharmacology, Tulane University Medical School,

New Orleans, Louisiana 70112, USA

e-mail: john.mclachlan@tulane.edu

1. Tham, D. M., Gardner, C. D. \& Haskell, W. L. J. Clin. Endocrinol. Metab. 83, 2223-2235 (1998).

2. Collins-Burow, B. M. et al. Nutr. Cancer 38, 229-244 (2000).

3. McLachlan, J. A. Endocrinol. Rev. 22, 319-341 (2001).

4. Schultze, M. \& Kondorosi, A. Annu. Rev. Genet. 32, 33-57 (1998).

5. Peters, N. K., Frost, J. W. \& Long, S. R. Science 233,
977-980 (1986).

6. Djordjevic, M. A. et al. EMBO J. 6, 1173-1179 (1987).

7. Firmin, J. L. et al. Nature 324, 90-93 (1986).

8. Gyorgypal, Z. \& Kondorosi, A. Mol. Gen. Genet. 226, 337-340 (1991).

9. Mulligan, J. T. \& Long, S. R. Proc. Natl Acad. Sci. USA 82, 6609-6613 (1985).

10. Peters, N. K. \& Long, S. R. Plant Physiol. 88, 396-400 (1988).

11. Redmond, J. W. et al. Nature 323, 632-635 (1986).

12. Gage, D. J., Bobo, T. \& Long, S. R. J. Bacteriol. 178, 7159-7166 (1996).

13. Baker, M. E. Adv. Exp. Med. Biol. 439, 249-276 (1998).

COMMUNICATIONS ARISING

Palaeovegetation

\section{Diversity of temperate plants in east Asia}

he exceptionally broad species diversity of vascular plant genera in east Asian temperate forests, compared with their sister taxa in North America, has been attributed to the greater climatic diversity of east Asia, combined with opportunities for allopatric speciation afforded by repeated fragmentation and coalescence of populations through Late Cenozoic ice-age cycles ${ }^{1}$. According to Qian and Ricklefs ${ }^{1}$, these opportunities occurred in east Asia because temperate forests extended across the continental shelf to link populations in China, Korea and
Japan during glacial periods, whereas higher sea levels during interglacial periods isolated these regions and warmer temperatures restricted temperate taxa to disjunct refuges. However, palaeovegetation data from east Asia $^{2-6}$ show that temperate forests were considerably less extensive than today during the Last Glacial Maximum, calling into question the coalescence of tree populations required by the hypothesis of Qian and Ricklefs ${ }^{1}$.

Pollen data ${ }^{2-6}$ from ${ }^{14} \mathrm{C}$-dated sediment cores, processed using a standard interpretive technique ${ }^{7}$, record the changes in vegetation between the Last Glacial Maximum (Fig. 1a) and the present (Fig. 1b). These data are derived from work carried out by Chinese and Japanese palynologists during the two decades since the reconstruction by CLIMAP cited in ref. 1 . The data indicate that temperate forests were restricted in distribution during the Last Glacial Maximum, a finding that is contrary to the inference by Qian and Ricklefs ${ }^{1}$. The forests were displaced either by boreal and mixed forests lacking most of the temperate forest taxa, or by treeless vegetation (steppe and desert, with pollen assemblages dominated by Artemisia, Chenopodiaceae and grasses), over much of their present range.

Low levels of precipitation, compounded by the low water-use efficiency of $\mathrm{C}_{3}$ photosynthesis at atmospheric $\mathrm{CO}_{2}$ concentrations during the Last Glacial Maximum (less than 200 p.p.m.; refs 7, 8), confined temperate forests to medium elevations in northern China, whereas low temperatures caused mixed forests (mixtures of boreal conifers with only the most cold-tolerant temperate taxa) to dominate in much of Japan and southern China ${ }^{2-6,8}$.

To provide a spatially continuous vegetation map (Fig. 1c) that is physically consistent with climate forcing for the Last Glacial Maximum, we used a model of climate in the last glacial maximum (based on known changes in insolation, atmospheric composition and physiography) ${ }^{9}$ as input to an equilibrium biogeography model, BIOME4 (Fig. 1d) ${ }^{10}$. The large-scale features of this simulation are supported by pollen data (Fig. 1a). Temperate forests are shown as confined to a relatively narrow belt at low elevations, whereas non-forest vegetation is shown to be greatly extended, as the pollen data indicate. On the other hand, a band of temperate deciduous forest is shown extending across the East China Sea, consistent both with Qian and Ricklefs' hypothesis ${ }^{1}$ and with pollen evidence for the existence of an offshore glacial refuge for temperate deciduous trees.

Quaternary palaeodata have repeatedly overturned historical biogeographical hypotheses that were based on presentday taxon distributions. The palaeodata indicate a more complex history in which 